\title{
Risk of cancer in regular and low meat-eaters, fish-eaters, and vegetarians: a prospective analysis of UK Biobank participants
}

Cody Z. Watling ${ }^{*}$ (D) Julie A. Schmidt, Yashvee Dunneram, Tammy Y. N. Tong, Rebecca K. Kelly, Anika Knuppel, Ruth C. Travis, Timothy J. Key and Aurora Perez-Cornago

\begin{abstract}
Background: Following a vegetarian diet has become increasingly popular and some evidence suggests that being vegetarian may be associated with a lower risk of cancer overall. However, for specific cancer sites, the evidence is limited. Our aim was to assess the associations of vegetarian and non-vegetarian diets with risks of all cancer, colorectal cancer, postmenopausal breast cancer, and prostate cancer and to explore the role of potential mediators between these associations.

Methods: We conducted a prospective analysis of 472,377 UK Biobank participants who were free from cancer at recruitment. Participants were categorised into regular meat-eaters ( $n=247,571)$, low meat-eaters $(n=205,385)$, fisheaters $(n=10,696)$, and vegetarians $(n=8685)$ based on dietary questions completed at recruitment. Multivariableadjusted Cox regressions were used to estimate hazard ratios (HR) and 95\% confidence intervals (Cl) for all cancer incidence and separate cancer sites across diet groups.
\end{abstract}

Results: After an average follow-up of 11.4years, 54,961 incident cancers were identified, including 5882 colorectal, 7537 postmenopausal breast, and 9501 prostate cancers. Compared with regular meat-eaters, being a low meat-eater, fish-eater, or vegetarian were all associated with a lower risk of all cancer (HR: 0.98, 95\% Cl: 0.96-1.00; 0.90, 0.84-0.96; $0.86,0.80-0.93$, respectively). Being a low meat-eater was associated with a lower risk of colorectal cancer in comparison to regular meat-eaters $(0.91,0.86-0.96)$; however, there was heterogeneity in this association by sex $(p=0.007)$, with an inverse association across diet groups in men, but not in women. Vegetarian postmenopausal women had a lower risk of breast cancer $(0.82,0.68-0.99)$, which was attenuated and non-significant after adjusting for body mass index (BMI; 0.87, 0.72-1.05); in mediation analyses, BMI was found to possibly mediate the observed association. In men, being a fish-eater or a vegetarian was associated with a lower risk of prostate cancer $(0.80,0.65-0.99$ and 0.69 , 0.54-0.89, respectively).

Conclusion: The lower risk of colorectal cancer in low meat-eaters is consistent with previous evidence suggesting an adverse impact of meat intake. The lower risk of postmenopausal breast cancer in vegetarian women may be explained by their lower BMI. It is not clear whether the other differences observed for all cancers and for prostate

*Correspondence: cody.watling@ndph.ox.ac.uk

Cancer Epidemiology Unit, Nuffield Department of Population Health,

University of Oxford, Oxford OX3 7LF, UK original author(s) and the source, provide a link to the Creative Commons licence, and indicate if changes were made. The images or other third party material in this article are included in the article's Creative Commons licence, unless indicated otherwise in a credit line to the material. If material is not included in the article's Creative Commons licence and your intended use is not permitted by statutory regulation or exceeds the permitted use, you will need to obtain permission directly from the copyright holder. To view a copy of this licence, visit http://creativecommons.org/licenses/by/4.0/. The Creative Commons Public Domain Dedication waiver (http://creativeco mmons.org/publicdomain/zero/1.0/) applies to the data made available in this article, unless otherwise stated in a credit line to the data. 
cancer reflect any causal relationships or are due to other factors such as residual confounding or differences in cancer detection.

Keywords: Diet, Vegetarian, Pescatarian, Colorectal, Breast, Prostate, Cohort, Meat

\section{Background}

Cancer is a leading cause of death worldwide [1], and in the United Kingdom (UK), 28\% of all deaths in 2017 were attributable to cancer [2-4]. Colorectal, breast, and prostate cancer collectively account for $39 \%$ of all new cancer diagnoses in the UK [5], and it has been estimated that nearly $40 \%$ of cancer cases may be preventable through modifiable factors [6, 7]. Although several dietary factors have been suggested to influence cancer risk, it remains unclear whether dietary patterns are related to the risk of developing cancer $[8,9]$.

It has been hypothesised that vegetarian diets, which exclude the consumption of all meat and fish, may be associated with a lower cancer risk. In addition to excluding red and processed meat, which are associated with an increased risk of colorectal cancer [8], vegetarians also generally consume higher amounts of plant foods such as fruits, vegetables, and whole grains compared to meateaters $[10,11]$, which might also contribute to lowering the risk of some site-specific cancers [8]. Evidence from two large cohorts which include a large proportion of vegetarians, the European Prospective Investigation into Cancer and Nutrition-Oxford (EPIC-Oxford) and the Adventist Health Study-2 (AHS-2), has suggested that vegetarians may have a lower risk of developing cancer (all types combined) in comparison to meat-eaters [12, 13], but the evidence remains unclear for individual cancer sites [12, 14-17]. Moreover, the risk of cancer in those who do not consume meat but do eat fish (fish-eaters or pescatarians) may differ from that of meat-eaters; some evidence has suggested that fish-eaters may have a lower overall risk of cancer [12], and a lower risk of colorectal cancer $[12,16]$ than meat-eaters, but no differences have been reported for breast [14] or prostate cancer risk [12, 15]. Despite the substantial number of vegetarians and fish-eaters in these cohorts (8000-25,000 participants), power to detect an association for specific cancer sites may be limited due to relatively small numbers of cancer cases ( 5000 total cases) in these individual studies [12, $14-17]$.

Any difference in cancer risk between diet groups may be due to differences in physiological characteristics, including adiposity. In western populations, vegetarians and fish-eaters have been shown to have lower body mass indices in comparison with the body mass index (BMI) of meat-eaters [18-20] which is important for cancer risk because obesity is a known risk factor for several cancer sites [8]. Another hypothesised explanation for the lower risk of cancer observed amongst vegetarians and fish-eaters is the possible differences in hormone levels [21], such as insulin-like growth factor-I (IGF-I) and testosterone, which may be related to their dietary intakes [21-23]. Hormone difference may be important as higher levels of IGF-I have been associated with higher risks of colorectal, breast, and prostate cancer [24] and higher levels of free testosterone have been associated with prostate cancer [25] and postmenopausal breast cancer [26].

To further understand these relationships, we assessed the associations of diet groups with risks of all, colorectal, postmenopausal breast, and prostate cancer in the UK Biobank, which includes approximately 10,000 fisheaters, 8000 vegetarians, and nearly 55,000 total incident cancer cases. We additionally aimed to assess the roles of BMI, circulating IGF-I, and calculated free testosterone as potential mediators of the observed associations between diet groups and cancer risk.

\section{Methods \\ Study design and participants}

Potential participants were first identified for the UK Biobank study using National Health Service (NHS) records, and 9.2 million eligible individuals, aged 40-70 and living within 25 miles of one of the assessment centres in the UK, were invited to participate in the study. Over 500,000 participants (5.5\% response rate) consented to participate between 2006 and 2010 [27] and visited one of 22 assessments centres across England, Wales, and Scotland. A full description of the study protocol can be found on the UK Biobank website [27].

The UK Biobank was approved by the NHS North West Multicentre Research Ethics Committee (21/NW/0157). All participants provided informed consent at recruitment, allowing for follow-up using data-linkage to health records. The study was performed in accordance to the Declaration of Helsinki.

\section{Exclusions}

Participants were excluded from this analysis if they withdrew consent over the study period $(n=871)$, had a prevalent cancer diagnosis at recruitment (excluding non-melanoma skin cancer International Statistical Classification of Disease (ICD-10) code: C44; $n=29,504)$, their genetic sex was different from their reported sex $(n=321)$, or they did not contribute any 
follow-up time ( $n=2$; Additional File 1 Fig. S1). Participants who responded as 'do not know' or 'prefer not to say' for all dietary questions regarding meat intake were also excluded from the analyses $(n=282)$. This left a total of 472,337 participants, of whom 217,937 were males and 254,400 were females. For prostate cancer analyses, women were excluded, and for postmenopausal breast cancer analyses, women who were premenopausal at recruitment and did not reach the age of 55 over the follow-up time $(n=16,222)$, and men, were excluded.

\section{Diet group classification}

Diet groups were categorised using the touchscreen questionnaire completed at recruitment which asked participants about their frequency of consumption of processed meat, beef, lamb or mutton, pork, chicken, turkey or other poultry, and oily and non-oily fish. Participants chose a frequency of intake ranging from "Never" to "Once or more daily". From these responses, participants were categorised into four diet groups (regular meateaters; low meat-eaters; fish-eaters; and vegetarians). Regular meat-eaters were participants who said they consumed processed, red meat (beef, pork, lamb), or poultry $>5$ times a week. Low meat-eaters were participants who reported consuming processed, red meat, or poultry $\leq 5$ times a week. Fish-eaters were participants who reported that they never consumed red meat, processed meat, or poultry but ate oily and/or non-oily fish. Vegetarians were defined as participants who reported that they never consumed any meat or fish. The vegetarian group also included vegans who reported not consuming any meat, fish, dairy, or eggs $(n=446)$.

\section{Covariates and biomarkers}

The baseline touchscreen questionnaire also asked participants about sociodemographic, reproductive, and lifestyle factors. In addition, all participants had their blood drawn and anthropometric measurements, including height and weight, taken by a trained professional. Further information on covariate data collection and classification can be found in the Additional File 1 Supplementary Methods.

Non-fasting blood samples were provided by $99.7 \%$ of participants at recruitment and were shipped to the central processing laboratory at $4{ }^{\circ} \mathrm{C}$ prior to serum preparation, aliquoting, and cryopreservation in the central working archive. Biochemistry markers were measured including insulin-like growth factor-I (IGF-I) and testosterone, as well as sex hormone-binding globulin which we used to calculate an estimate of free testosterone [28]. Further description of the UK Biobank biomarker measurements can be found online [29].

\section{Follow-up and outcome ascertainment}

Data on cancer diagnosis were ascertained using a combination of records from the NHS Digital (cancer registry) and Public Health England for participants from England and Wales, NHS Central Register for participants from Scotland [30] as well as the Hospital Episodes Statistics (HES) data for English participants and Scottish Morbidity Records (SMR) for Scottish participants (please see details in the Additional File 1 Supplementary Methods). Using the World Health Organization's ICD10 codes, participants were classified as having an event if they had an incident diagnosis of cancer recorded as: all cancer (C00-97 excluding non-melanoma skin cancer: C44), colorectal cancer (C18-C20), breast cancer (C50), or prostate cancer (C61), or if no prior incident diagnosis was reported their primary underlying cause of death was the respective cancer. Participants contributed follow-up time from the date of recruitment until the date of the first cancer registration or cancer first recorded on death certificate, date of death, or last day of follow-up available from HES and SMR data (28 February 2021 for England and Scotland). Cancer registry data were available until 31 July 2019 for England and Wales, and 31 October 2015 for Scotland; after this time, only HES and SMR data were used for the follow-up of participants. For Welsh participants, hospital episode data did not extend past the cancer registry censoring date and therefore were not used. For breast cancer, analyses were restricted to postmenopausal breast cancer and women contributed follow-up time beginning when they turned 55 years of age or their date at recruitment if they were categorised as being postmenopausal from questions asked at baseline (see Additional File 1 Supplementary Methods for further details) [31].

\section{Statistical analyses}

Baseline characteristics of UK Biobank participants were summarised across diet groups for all participants, and separately for men and women.

Cox proportional hazards regressions were used, with age as the underlying time variable, to estimate hazard ratios (HR) and 95\% confidence intervals (CI). Minimally adjusted models were stratified by sex (for all cancer and colorectal cancer analyses only) and age at recruitment ( $<45,45-49,50-54,55-59,60-64, \geq 65$ years) and adjusted for region at recruitment (North-West England, North-Eastern England, Yorkshire \& the Humber, West Midlands, East Midlands, South-East England, SouthWest England, London, Wales, and Scotland).

Multivariable-adjusted Cox regression models for all analyses were further adjusted for height (eight sexspecific categories increasing by $5 \mathrm{~cm}$, and unknown/ missing $(0.51 \%)$ ), physical activity (low: 0-9.99, medium: 
10-49.99, high: $\geq 50$ metabolic equivalent of task-hours /week, and unknown/missing (4.04\%)), Townsend deprivation index (quintiles from most deprived to least deprived, and unknown/missing $(0.13 \%)$ ), education (completion of national exam at age 16, completion of national exam at age 17-18, college or university degree, or other/unknown/missing (18.7\%)), employment status (employed, retired, not in paid employment, or unknown $(1.15 \%)$ ), smoking status (never, former, light smoker: $\leq$ 15 cigarettes/day, medium smoker: 16-29 cigarettes/ day, heavy smoker: $\geq 30$ cigarettes/day, or missing/ unknown $(0.65 \%)$ ), alcohol consumption (none drinkers, $<1,1-9.99,10-19.99, \geq 20 \mathrm{~g} /$ day, or unknown/missing $(0.73 \%)$ ), ethnicity (White, Mixed race or other, Asian or British Asian, and Black or Black British, or missing/unknown $(0.56 \%)$ ), and diabetes status (no, yes, or unknown (0.53\%)).

For colorectal cancer and for all cancer sites, multivariable models were further adjusted for female specific covariates: menopausal hormone therapy (MHT) use (no, former, current, or unknown $(0.58 \%)$ ) and menopausal status at recruitment (premenopausal, postmenopausal, or unknown (9.0\%)). Moreover, for colorectal cancer, multivariable models were adjusted for non-steroidal anti-inflammatory drug use (NSAID; no reported use, irregular use, regular use of aspirin/ibuprofen). For prostate cancer, models were additionally adjusted for marital status (not living with a partner, living with a partner) [32]. For postmenopausal breast cancer, models were additionally adjusted for MHT use (same as above), age at menarche ( $\leq 12$ years, 13 years old, $\geq$ 14 years, or unknown (22.5\%)), parity and age at first birth (nulliparous, 1-2 children <25years old, $3+$ children $<25$ years old, 1-2 children 25-29.9years old, 3+ children 25-29.9years old, 1-2 children $30+$ years old, $3+$ children $30+$ years old, or missing $(0.3 \%))$. Further information on covariate classification can be found in Additional File 1 Supplementary Methods. In all models, the proportional hazards assumption was evaluated using Schoenfeld residuals, and no violations were observed.

We considered BMI as a potential confounder as well as a mediator. When BMI was considered as a potential confounder, BMI measured at recruitment was added to multivariable models (multivariable adjusted + BMI; <20, 20-22.49, 22.5-24.9, 25.0-27.49, 27.5-29.9, $30-32.49, \quad 32.5-34.9, \geq 35 \mathrm{~kg} / \mathrm{m}^{2}$, or unknown $/ \mathrm{miss}-$ ing (0.57\%)). Models assessing BMI as a mediator are explained below in the mediation analyses section.

To determine if there was heterogeneity in the associations of diet groups with cancer risk, and to assess the influence of confounder adjustments [33, 34], $X^{2}$ statistics and $p$-values for including the diet group in the model were estimated using likelihood ratio tests (LRT) comparing a model without the diet groups variable to the model with the diet groups variable.

\section{Subgroup and sensitivity analyses}

For all analyses, we assessed heterogeneity by subgroups of BMI (median: $<27.5$ and $\geq 27.5 \mathrm{~kg} / \mathrm{m}^{2}$ ) and smoking status (ever and never) by using a LRT comparing the main model to a model including an interaction term between diet groups and the subgroup variable (BMI and smoking status). For colorectal cancer, we further assessed heterogeneity by sex. For all cancer sites combined, we additionally explored heterogeneity by smoking status, censoring participants at baseline who were diagnosed with lung cancer.

In sensitivity analyses, we excluded cases and participants who had less than 2 years of follow-up and all participants with missing data on covariates. We also examined associations separately in white participants because a large proportion of the vegetarians in this cohort are of South Asian ethnicity ( 17.5\%). Furthermore, we additionally adjusted for fruit and vegetable intake in the multivariable adjusted model ( $<3$ servings/ day, 3-3.99 servings/day, 4-5.99 servings/day, $\geq 6$ servings/day, unknown) to control for this component of dietary intake as a proxy for a healthy diet. For prostate cancer analyses, we included in the multivariable adjusted model prostate-specific antigen (PSA) testing (no PSA testing, had PSA test, or unknown) reported at baseline in all men and during follow-up from general practice records in a subsample $(n=99,412$ males; records available for participants until 31 May 2016 for England, 31 March 2017 for Scotland, and 31 August 2017 for Wales).

\section{Mediation analyses}

If a significant association was observed between a diet group and a cancer outcome in the main analyses, we then further explored potential mediators that have been shown to be associated or possibly associated with diet groups $[19,21]$ and were previously related to the cancer site of interest (BMI, IGF-I, and free testosterone) $[25,26]$. To determine if differences in mediators were observed by diet group, we used multivariable linear regression to compare the selected biomarker measurements (IGF-I and free testosterone [28]) and BMI across dietary groups, adjusting for potential confounders (see Additional File 1 Supplementary Methods). We did not explore mediation if there was no significant difference in cancer risk between each diet group and regular meat-eaters or if the biomarker concentrations were not significantly different between diet groups. We explored mediation via BMI for all cancer, colorectal cancer, and postmenopausal breast cancer risk [8], but 
not for prostate cancer due to its heterogeneous association with risk by stage and grade [35] and as these data are not available in this cohort. For prostate cancer and postmenopausal breast cancer, we also explored potential mediation via circulating concentrations of IGF-I and calculated free testosterone [25, 26, 28]. We did not explore biomarker mediation for the all cancer-diet group associations as these biomarkers have not been associated with all cancer risk.

To assess for mediation, we used the inverse odds ratio weighting (IORW) method [36, 37]. This method aims to decompose associations between diet group mediated by the potential mediator (natural indirect effect [NIE]) and the estimated association of diet group with cancer risk not mediated by baseline BMI or biomarkers (natural direct effect [NDE]). The term "effect" is used here in concordance with the causal mediation literature but should not be interpreted as implying causality. To determine the proportion of the association between diet groups and cancer outcome mediated by the mediator of interest (e.g. BMI), we took the log of the indirect effect HR and divided it by the log of the total effect HR. Further details of the mediation analyses can be found in the Additional File 1 Supplementary Methods [38, 39].

All analyses were conducted using Stata version 17.0 (Stata Corp LP, College Station, TX). P-values were two-sided with $p<0.05$ being considered statistically significant.

\section{Results}

Of the participants included in the analysis, 247,571 (52.4\%) were classified as regular meat-eaters, 205,385 (43.5\%) were low meat-eaters, 10,696 (2.3\%) were fisheaters and $8685(1.8 \%)$ were vegetarians. After an average of 11.4 years of follow-up, 54,961 incident cases of any type of cancer were diagnosed; 5882 participants were diagnosed with colorectal cancer, 7537 women were diagnosed with postmenopausal breast cancer, and 9501 men were diagnosed with prostate cancer.

Table 1 presents participants' baseline characteristics across diet groups. Vegetarians and fish-eaters had a lower BMI, were younger, more likely to be never smokers, have a university/college degree, and report consuming less alcohol at recruitment compared to regular meat-eaters. Vegetarian men were also less likely to have had a PSA test in comparison to meat-eaters (Table 1). Additional File 1 Table S1 presents the baseline characteristics across diet groups stratified by sex. Both men and women fish-eaters and vegetarians had lower BMIs and were younger at recruitment in comparison to regular meat-eaters.

The minimally adjusted models and sequential adjustments for the associations between diet groups and cancer risks are presented in Additional file 1 Table S2, and Fig. 1 shows the multivariable-adjusted models. In the multivariable-adjusted models (not including BMI), a vegetarian diet was associated with a lower risk of all cancer (HR: 0.86, 95\% CI: 0.80-0.93), postmenopausal breast cancer $(0.82,0.68-0.99)$, and prostate cancer $(0.69$, 0.54-0.89; Fig. $1 \mathrm{~A}$ ) in comparison to regular meat-eaters. Furthermore, compared to being a regular meat-eater, fish-eaters had a lower risk of all cancers $(0.90,0.84-0.96)$ and prostate cancer $(0.80,0.65-0.99)$, and low meat-eaters had a lower risk of colorectal cancer $(0.91,0.86-0.96$; Fig. 1A). When including BMI as a potential confounder, associations were slightly attenuated apart from prostate cancer which did not change (Fig. 1B). For postmenopausal breast cancer, after adjustment for BMI, the risk for vegetarians compared to regular meat-eaters was no longer statistically significant $(0.87,0.72-1.05$; Fig. 1B).

\section{Subgroup analyses}

No evidence of heterogeneity was observed across BMI subgroups in the associations between diet groups and risk of all cancer, colorectal, postmenopausal breast, and prostate cancer (Additional File 1 Tables S3-S6). For colorectal cancer, there was evidence of heterogeneity by sex $\left(P_{\text {het }}=0.007\right)$, with male low meat-eaters, fish-eaters, and vegetarians having a lower risk of colorectal cancer $(0.89,0.83-0.95 ; 0.69,0.47-1.01 ; 0.57,0.36-0.91$, respectively) in comparison to regular meat-eaters, whereas no significant association was observed across diet groups for females (Additional File 1 Table S4). For smoking status, some evidence of heterogeneity was observed in the association between diet groups and all cancer risk $\left(p_{\text {het }}=0.056\right)$; amongst ever smokers, the low meateaters, fish-eaters, and vegetarians had lower risks of all cancer sites than regular meat-eaters $(0.97,0.94-0.99$; $0.86,0.78-0.95 ; 0.79,0.70-0.90$, respectively), whereas these associations were non-significant for non-smokers (Additional File 1 Table S3). However, when censoring participants who developed lung cancer during followup, the test for heterogeneity by smoking status became non-significant between diet groups $\left(p_{\text {het }}=0.223\right.$; Additional File 1 Table S3) although the association with diet group for all cancer sites was still only significant amongst ever smokers.

\section{Sensitivity analyses}

Associations remained largely the same when analyses were restricted to participants of white European ancestry and when participants with missing data were excluded (Additional File 1 Fig. S2). When the participants who had an event or were censored in the first 2 years of follow-up were excluded, results remained mostly the same except that being a fish-eater was more 
Table 1 Baseline characteristics across diet groups in UK Biobank

\begin{tabular}{|c|c|c|c|c|}
\hline & \multicolumn{4}{|l|}{ Diet groups } \\
\hline & Regular meat-eaters & Low meat-eaters & Fish-eaters & Vegetarians \\
\hline Number of participants & 247,571 & 205,385 & 10,696 & 8685 \\
\hline Age at recruitment-years, mean (SD) & $56.0(8.2)$ & $56.9(8.0)$ & $54.0(8.0)$ & $53.0(7.9)$ \\
\hline Sex-female & $114,849(46.4 \%)$ & $126,165(61.4 \%)$ & $7664(71.7 \%)$ & $5722(65.9 \%)$ \\
\hline $\mathrm{BMI}-\mathrm{kg} / \mathrm{m}^{2}$, mean (SD) & $27.9(4.9)$ & $27.0(4.7)$ & $25.3(4.3)$ & $25.7(4.7)$ \\
\hline Male height—cm, mean (SD) & $175.7(6.8)$ & $175.4(6.9)$ & $176.4(6.9)$ & $175.5(7.2)$ \\
\hline Female height—cm, mean (SD) & $162.4(6.3)$ & $162.5(6.3)$ & $163.5(6.4)$ & $162.1(6.8)$ \\
\hline \multicolumn{5}{|l|}{ Physical activity } \\
\hline Low & $72,811(29.4 \%)$ & $59,752(29.1 \%)$ & $2430(22.7 \%)$ & $2371(27.3 \%)$ \\
\hline Moderate & $116,591(47.1 \%)$ & $98,692(48.1 \%)$ & $5710(53.4 \%)$ & $4346(50.0 \%)$ \\
\hline High & $48,012(19.4 \%)$ & $38,704(18.8 \%)$ & $2273(21.3 \%)$ & $1690(19.5 \%)$ \\
\hline \multicolumn{5}{|l|}{ Townsend deprivation index } \\
\hline Q1-Most affluent & $51,117(20.6 \%)$ & $40,433(19.7 \%)$ & $1777(16.6 \%)$ & $1258(14.5 \%)$ \\
\hline Q5-Most deprived & $48,227(19.5 \%)$ & $41,627(20.3 \%)$ & $2385(22.3 \%)$ & $2216(25.5 \%)$ \\
\hline \multicolumn{5}{|l|}{ Ethnicity } \\
\hline White & $233,959(94.5 \%)$ & $193,033(94.0 \%)$ & $9922(92.8 \%)$ & $6903(79.5 \%)$ \\
\hline Mixed other & $3576(1.4 \%)$ & $3294(1.6 \%)$ & $172(1.6 \%)$ & $152(1.8 \%)$ \\
\hline Asian or British Asian & $4114(1.7 \%)$ & $5054(2.5 \%)$ & $369(3.4 \%)$ & $1524(17.5 \%)$ \\
\hline Black or Black British & $4218(1.7 \%)$ & $3295(1.6 \%)$ & $167(1.6 \%)$ & $48(0.6 \%)$ \\
\hline \multicolumn{5}{|l|}{ Education } \\
\hline 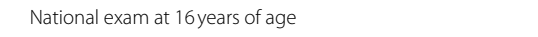 & $41,764(16.9 \%)$ & $34,271(16.7 \%)$ & $1180(11.0 \%)$ & $1099(12.7 \%)$ \\
\hline National exam at $17-18$ years of age & $13,750(5.6 \%)$ & $10,805(5.3 \%)$ & $578(5.4 \%)$ & $551(6.3 \%)$ \\
\hline Degree or college & $146,214(59.1 \%)$ & $119,791(58.3 \%)$ & $8015(74.9 \%)$ & $6109(70.3 \%)$ \\
\hline \multicolumn{5}{|l|}{ Employment } \\
\hline In paid employment & $146,078(59.0 \%)$ & $115,579(56.3 \%)$ & $7338(68.6 \%)$ & $6065(69.8 \%)$ \\
\hline Retired & $77,483(31.3 \%)$ & $70,640(34.4 \%)$ & $2341(21.9 \%)$ & $1582(18.2 \%)$ \\
\hline Not in paid employment & $21,068(8.5 \%)$ & $17,028(8.3 \%)$ & $877(8.2 \%)$ & $921(10.6 \%)$ \\
\hline Living with a partner-yes & $187,545(75.8 \%)$ & $141,711(69.0 \%)$ & $6930(64.8 \%)$ & $5771(66.4 \%)$ \\
\hline \multicolumn{5}{|l|}{ Smoking status } \\
\hline Never & $132,294(53.4 \%)$ & $114,385(55.7 \%)$ & $6075(56.8 \%)$ & $5561(64.0 \%)$ \\
\hline Previous & $85,319(34.5 \%)$ & $69,642(33.9 \%)$ & $3800(35.5 \%)$ & $2480(28.6 \%)$ \\
\hline Light smoker < 15 cig/day & $7594(3.1 \%)$ & $6299(3.1 \%)$ & $290(2.7 \%)$ & $210(2.4 \%)$ \\
\hline Medium smoker 15-29 cig/day & $10,101(4.1 \%)$ & $6644(3.2 \%)$ & $157(1.5 \%)$ & $139(1.6 \%)$ \\
\hline Heavy smoker $30+$ cig/day & $10,418(4.2 \%)$ & $7432(3.6 \%)$ & $333(3.1 \%)$ & $252(2.9 \%)$ \\
\hline Alcohol intake g/day, mean (SD) & $19.9(21.3)$ & $14.9(16.6)$ & $13.6(14.5)$ & $13.0(16.1)$ \\
\hline Diabetic-yes & $15,603(6.3 \%)$ & $10,748(5.2 \%)$ & $290(2.7 \%)$ & $465(5.4 \%)$ \\
\hline $\begin{array}{l}\text { Prostate-specific antigen test reported at baseline or in follow- } \\
\text { up-yes, male only }\end{array}$ & $51,555(38.8 \%)$ & $33,394(42.2 \%)$ & $1125(37.1 \%)$ & $929(31.4 \%)$ \\
\hline \multicolumn{5}{|l|}{ Female specific covariates } \\
\hline Age at menarche-years, mean (SD) & $12.6(2.8)$ & $12.5(2.9)$ & $12.5(2.9)$ & $12.4(3.3)$ \\
\hline \multicolumn{5}{|l|}{ Menopausal status } \\
\hline Premenopausal & $24,939(21.7 \%)$ & $23,360(18.5 \%)$ & $2232(29.1 \%)$ & $1843(32.2 \%)$ \\
\hline Postmenopausal & $78,626(68.5 \%)$ & $92,413(73.2 \%)$ & $4717(61.5 \%)$ & $3342(58.4 \%)$ \\
\hline \multicolumn{5}{|l|}{ Menopausal hormone therapy use } \\
\hline Never & $70,830(61.7 \%)$ & $76,747(60.8 \%)$ & $5468(71.3 \%)$ & $4386(76.7 \%)$ \\
\hline Former & $34,149(29.7 \%)$ & $38,960(30.9 \%)$ & $1590(20.7 \%)$ & $9,66(16.9 \%)$ \\
\hline Current & $8993(7.8 \%)$ & $9988(7.9 \%)$ & $584(7.6 \%)$ & $320(5.6 \%)$ \\
\hline \multicolumn{5}{|l|}{ Parity } \\
\hline Nulliparous & $17,671(15.4 \%)$ & $25,569(20.3 \%)$ & $2330(30.4 \%)$ & $1736(30.3 \%)$ \\
\hline $1-2$ children & $67,306(58.6 \%)$ & $70,915(56.2 \%)$ & $3910(51.0 \%)$ & $2770(48.4 \%)$ \\
\hline $3+$ children & $29,343(25.5 \%)$ & $29,526(23.4 \%)$ & $1415(18.5 \%)$ & $1203(21.0 \%)$ \\
\hline Age at first birth—years, mean (SD) & $25.4(4.6)$ & $25.2(4.6)$ & $26.4(5.1)$ & $26.0(4.9)$ \\
\hline
\end{tabular}

Percentages include missing values and therefore may not add up to $100 \%$ 


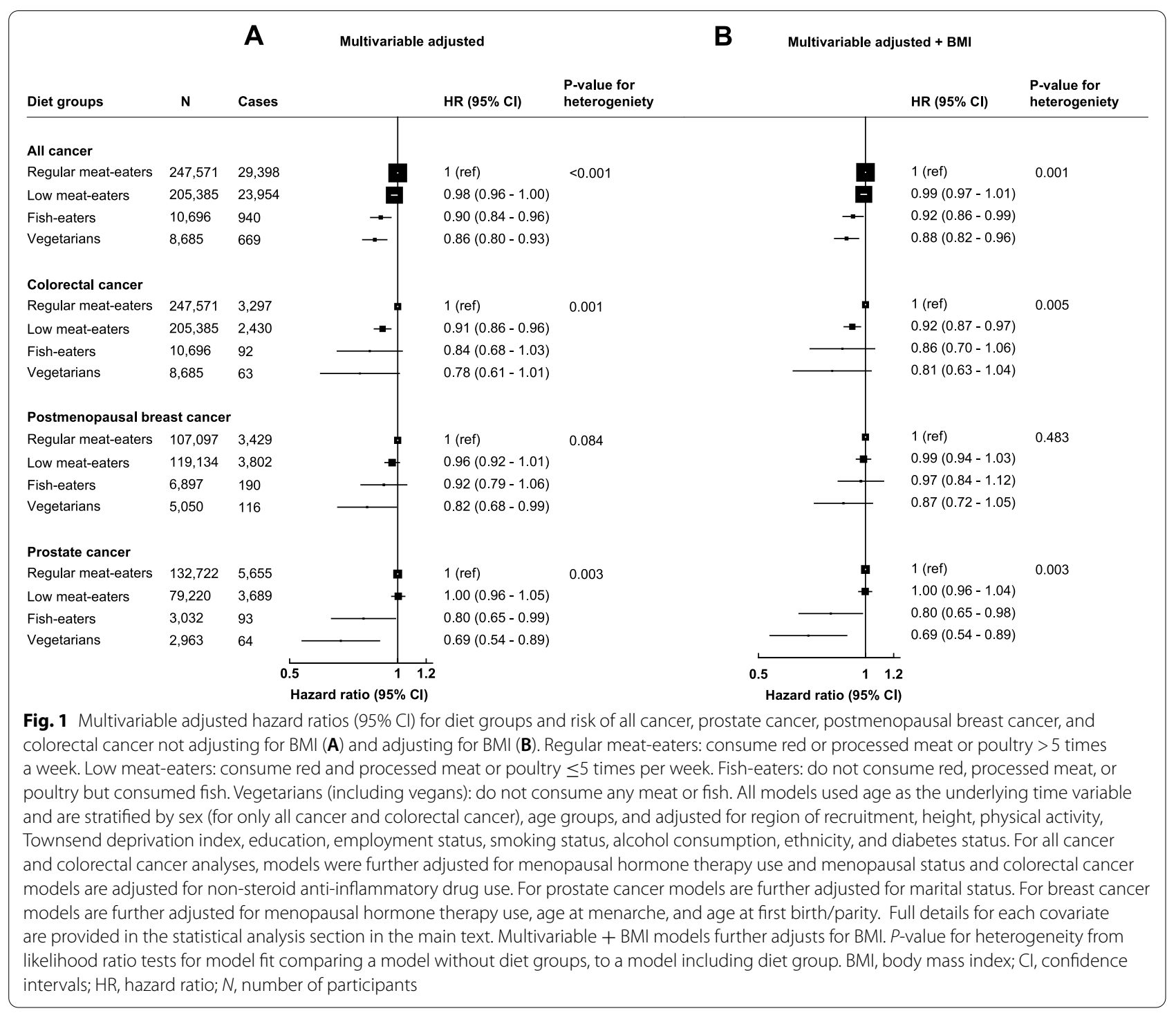

strongly associated with a lower risk of prostate cancer (HR 0.69, 0.55-0.88) in comparison to regular meateaters (Additional File 1 Fig. S3). In analyses, additionally adjusted for intake of fruit and vegetables in the multivariable models, no changes in associations were observed (Additional File 1 Fig. S3). For prostate cancer risk, when PSA testing was added to multivariable models, the associations were not materially changed (Additional File 1 Table S2).

\section{Mediation analyses}

Adjusted and relative means of BMI, IGF-I, and free testosterone across diet groups are shown in Additional File 1 Table S7. Explorations of potential mediators for significant diet-cancer associations are shown in Table 2. When we considered the potential of mediation via $\mathrm{BMI}$ in the associations of diet groups and risk of all cancer, this was not found to substantially mediate the observed associations (Table 2). For colorectal cancer risk, BMI was not found to mediate the observed lower risk in low meat-eaters compared with regular meat-eaters (Table 2); hormonal biomarkers were not explored because no differences in concentrations were observed between regular and low meat-eaters (Additional File 1 Table S7). For postmenopausal breast cancer risk, BMI was found to be a potential mediator for the observed difference in the risk between vegetarians and regular meat-eaters, with a decomposed $\mathrm{HR}^{\mathrm{NIE}}$ of 0.83 (95\% CI: 0.63-1.08) implying that BMI may explain nearly $93 \%$ of the lower risk observed in vegetarian women although this was not statistically significant (Table 2). When IGF-I was explored independently and after adjusting for BMI, a $\mathrm{HR}^{\mathrm{NIE}}$ of 0.91 (95\% CI: 0.731.15) was observed. For prostate cancer risk, IGF-I and 
Table 2 Summary of estimated direct effect, indirect effect, and total effect using potential mediators for the association of diet groups in comparison to regular meat-eaters and risk of all cancer, colorectal cancer, postmenopausal breast cancer, and prostate cancer risk

\begin{tabular}{|c|c|c|c|}
\hline \multirow[b]{2}{*}{ All cancer } & \multicolumn{3}{|c|}{ Potential mediators (hazard ratio; $95 \% \mathrm{Cl}$ ) } \\
\hline & Mediation through BMI & Mediation through IGF-I ${ }^{\mathrm{a}}$ & Mediation through free testosterone $\mathrm{a}^{\mathrm{a}}$ \\
\hline Low meat-eaters versus regular meat-eaters & Mediation through BMI $(n=450,412)$ & & \\
\hline Total effect & $0.99(0.96-1.00)$ & & \\
\hline Natural indirect effect & $0.99(0.98-1.00)$ & & \\
\hline \multirow[t]{2}{*}{ Natural direct effect } & $0.99(0.95-1.00)$ & & \\
\hline & Mediation through BMI $(n=256,727)$ & & \\
\hline \multicolumn{4}{|l|}{ Fish-eaters versus regular meat-eaters } \\
\hline Total effect & $0.90(0.83-0.97)$ & & \\
\hline Natural indirect effect & $0.99(0.90-1.01)$ & & \\
\hline \multirow[t]{2}{*}{ Natural direct effect } & $0.90(0.83-0.98)$ & & \\
\hline & Mediation through BMI $(n=254,709)$ & & \\
\hline \multicolumn{4}{|l|}{ Vegetarians versus regular meat-eaters } \\
\hline Total effect & $0.86(0.78-0.96)$ & & \\
\hline Natural indirect effect & $0.94(0.81-1.08)$ & & \\
\hline Natural direct effect & $0.92(0.77-1.09)$ & & \\
\hline Colorectal cancer & Mediation through BMI $(n=450,412)$ & & \\
\hline \multicolumn{4}{|l|}{ Low meat-eaters versus regular meat-eaters } \\
\hline Total effect & $0.91(0.85-0.97)$ & & \\
\hline Natural indirect effect & $1.00(0.98-1.02)$ & & \\
\hline Natural direct effect & $0.91(0.86-0.97)$ & & \\
\hline Postmenopausal breast cancer & Mediation through BMI $(n=111,574)$ & Mediation through IGF-I $(n=103,853)$ & $\begin{array}{l}\text { Mediation through free testosterone } \\
(n=93,662)\end{array}$ \\
\hline \multicolumn{4}{|l|}{ Vegetarians versus regular meat-eaters } \\
\hline Total effect & $0.82(0.68-0.99)$ & $0.86(0.71-1.05)$ & $0.86(0.71-1.05)$ \\
\hline Natural indirect effect & $0.83(0.63-1.08)$ & $0.91(0.73-1.15)$ & $1.06(0.76-1.38)$ \\
\hline Natural direct effect & $0.99(0.79-1.23)$ & $0.94(0.70-1.21)$ & $0.81(0.62-1.05)$ \\
\hline Prostate cancer ${ }^{b}$ & & Mediation through IGF-I $(n=126,538)$ & $\begin{array}{l}\text { Mediation through free testosterone } \\
(n=116,087)\end{array}$ \\
\hline \multicolumn{4}{|l|}{ Vegetarians versus regular meat-eaters } \\
\hline Total effect & & $0.71(0.56-0.92)$ & $0.71(0.56-0.92)$ \\
\hline Natural indirect effect & & $1.10(0.77-1.56)$ & $0.99(0.67-1.48)$ \\
\hline Natural direct effect & & $0.64(0.50-1.01)$ & $0.71(0.51-1.01)$ \\
\hline Fish-eaters versus regular meat-eaters & & & $\begin{array}{l}\text { Mediation through free testosterone } \\
(n=116,186)\end{array}$ \\
\hline Total effect & & & $0.80(0.65-0.99)$ \\
\hline Natural indirect effect & & & $0.95(0.70-1.29)$ \\
\hline Natural direct effect & & & $0.86(0.56-1.32)$ \\
\hline
\end{tabular}

All models used age as the underlying time variable and are stratified by sex (for only all cancer and colorectal cancer) and age groups at recruitment, and adjusted for region of recruitment, height, physical activity, Townsend deprivation index, education, employment status, smoking status, alcohol consumption, ethnicity, diabetes status, and body mass index (except when it was considered a potential mediator). For all cancer and colorectal cancer, models are further adjusted for menopausal hormone therapy use and menopausal status. Colorectal cancer models are adjusted for non-steroid anti-inflammatory drug use. For prostate cancer, models are further adjusted for marital status. For breast cancer, models are further adjusted for menopausal hormone therapy use, age at menarche, and age at first birth/ parity. Full details for each covariate are provided in the statistical analysis section in the main text.

Mediation analyses restricted to significant associations between diet-cancer in the main analyses (Fig. 1) and if there was a significant difference in biomarker concentrations between diet group (Additional File 1 Table S7).

Natural indirect effect represents the estimated association of diet group and cancer outcome through the potential mediator

Natural direct effect represents the estimated association of diet group and cancer outcome not through the potential mediator

Models exclude participants with missing values for mediator(s)

${ }^{\text {a }}$ Models are adjusted for BMI

${ }^{b}$ BMI not assessed as a mediator with total prostate cancer risk. Association of IGF-I and free testosterone presented as both hormones have been associated with prostate cancer risk. IGF-I concentrations not assessed for fish-eaters as no difference in concentrations in comparison to regular meat-eaters was observed. 
free testosterone concentrations did not seem to mediate the observed difference in risk between vegetarians and regular meat-eaters, and free testosterone was not found to mediate the difference in risk between fish-eaters and regular meat-eaters (Table 2).

\section{Discussion}

In this large British cohort, being a low meat-eater, fisheater, or vegetarian was associated with a lower risk of all cancer sites when compared to regular meat-eaters. We also found a lower risk of colorectal cancer amongst low meat-eaters, a lower risk of postmenopausal breast cancer risk in vegetarian women, and a lower risk of prostate cancer amongst vegetarian men. The lower risk of postmenopausal breast cancer in vegetarians may be largely a result of vegetarians having a lower BMI than regular meat-eaters, with possibly some further impact due to vegetarian women in this population having slightly lower circulating IGF-I concentrations.

\section{All cancer}

In this study, vegetarians, fish-eaters, and low meat-eaters all had a lower risk of developing all cancer in comparison to regular meat-eaters. It is important to consider that although some cancers may have similar aetiologies, some cancer sites may not be associated with dietary or nutritional factors and that using all cancer incidence as an outcome may crudely capture other lifestyle factors, outside of diet, that may be associated with cancer risk and may confound the associations observed; therefore, these results should be interpreted carefully. In the two largest previous prospective studies following vegetarians, EPIC-Oxford and AHS-2 found that being a vegetarian was associated with a $10 \%$ and $8 \%$ lower risk of all cancer than being a meat-eater, respectively, after adjusting for lifestyle risk factors and BMI [12, 13]. Fish-eaters in EPIC-Oxford had a lower risk of developing all cancer [12], but no association with risk for all cancer was observed for fish-eaters in comparison to meat-eaters in AHS-2 [13]. In the current analysis, we observed some evidence of heterogeneity by smoking status, and when we removed lung cancer from all cancer cases, significant associations were only observed across diet groups within the ever smoker subgroup. Therefore, the differences observed between diet groups for all cancer outcomes combined may not be due to diet and might be due to residual confounding by differences in other lifestyle factors, such as smoking.

\section{Colorectal cancer}

The risk of colorectal cancer was lower in low meat-eaters in comparison to regular meat-eaters whereas there was no significant difference for fish-eaters and vegetarians, potentially due to lack of power as the point estimates suggested lower risks in both these non-meat-eating diet groups. In both EPIC-Oxford and AHS-2, being a fisheater was associated with a lower risk of colorectal cancer in comparison with meat-eaters, whereas no association was observed for being vegetarian and risk of colorectal cancer compared to regular meat-eaters $[12,16]$. We also observed heterogeneity by sex, in that significant inverse associations were observed with risk across diet groups in men, when compared to regular meat-eaters, but not for women. This may in part be due to dietary differences between sexes; however, the number of colorectal cancer cases in some diet groups was too small to draw a clear conclusion. The intake of processed meat has been evaluated by the World Health Organization and World Cancer Research Fund to be a definite cause of colorectal cancer [40] and red meat as a probable cause of colorectal cancer $[40,41]$. This is likely to at least in part explain the lower risk of colorectal cancer in low meat-eaters, and mechanisms suggested include chemicals in meat such as nitrosamines [40, 42]. Overweight and obesity also increase the risk for colorectal cancer [43, 44], but in mediation analyses, BMI did not appear to mediate the difference observed between low meat-eaters and regular meat-eaters.

\section{Postmenopausal breast cancer}

A borderline significantly lower risk for postmenopausal breast cancer was observed for vegetarian women, which appeared to be largely due to their lower BMI as evidenced in mediation analyses and the attenuation of estimates when analyses were adjusted for BMI. We also observed a small potential effect for mediation for lower risk of postmenopausal breast cancer for vegetarians through lower IGF-I concentrations, perhaps influenced by the inclusion of vegans in this group [23]. To date, studies have reported a non-significantly lower risk of breast cancer for women following a vegetarian or pescatarian diet with or without adjustment for BMI [12, $14,17,45]$, which may be due to lack of power to detect modest associations in individual studies. Breast cancer is a heterogeneous disease, with differing risk factors by menopausal status and hormone receptor status [46]. $\mathrm{BMI}$ is robustly associated with higher postmenopausal breast cancer risk, probably due to higher circulating oestrogen derived from aromatisation of androgens in the adipose tissue [46]. As such, being vegetarian would be expected to confer a lower risk of postmenopausal breast cancer in comparison to meat-eaters because vegetarians generally have a lower BMI, but whether BMI is a confounder or a mediator for this association is not clear; if vegetarians have a lower BMI because of their diet then BMI may be considered a mediator, but if vegetarians 
have a lower BMI that is not due to their dietary intake but rather due to other non-dietary lifestyle factors (e.g. physical activity), then BMI would be considered to be a confounder.

Previous research has also suggested that vegetarian women are less likely to use MHT or to attend breast cancer screening [47]. In this analysis, we adjusted for MHT use at baseline, but residual confounding due to differences in use of MHT during follow-up is still possible. Data on breast cancer screening during follow-up were not available in this cohort; therefore, full adjustment for screening attendance was not possible and differences between diet groups in screening may have influenced our findings.

\section{Prostate cancer}

The risk of prostate cancer was lower in men who were vegetarians or fish-eaters in comparison to regular meat-eaters, but no difference in risk was observed for low meat-eaters. Previous analyses in the EPIC-Oxford cohort found a non-significantly lower risk of prostate cancer for British vegetarians and fish-eaters in comparison to meat-eaters [12]. In the AHS-2 study, no difference was found for vegetarians or fish-eaters, whereas being vegan was associated with a $35 \%$ lower risk of prostate cancer (based on 1079 cases in the cohort of which only 59 were in vegans) [15]. To date, no established dietary risk factor has been found in relation to prostate cancer risk, although there is some evidence which suggests that higher intake of dairy products, and possibly milk specifically, may increase the risk of prostate cancer [48]. This association has been proposed to be possibly mediated through IGF-I [22, 49], a hormone shown to be positively associated with both milk intake and prostate cancer risk $[25,50]$. In this cohort, slightly lower IGF-I concentrations have been observed in vegetarians compared to regular meat-eaters [21], and IGF-I has also been associated with prostate cancer risk [25]; however, the difference in IGF-I concentrations between these diet groups is small and may not confer a substantial difference in prostate cancer risk. As might be expected, in mediation analyses, the estimates were imprecise and there was no evidence that the difference in IGF-I concentrations between diet groups mediates the observed associations with cancer risk.

In this cohort, vegetarian men were less likely than meat-eaters to have had a PSA screening test at recruitment; therefore, vegetarians may have a lower risk of having prostate cancer diagnosed following a PSA test. Similarly, two other cohorts have also reported that vegetarian men were less likely to have had a PSA test [47, 51]. When data at recruitment and available general practice records during follow-up were assessed for PSA testing in the UK Biobank, there was only a small difference with $40 \%$ of regular meat-eaters and $37 \%$ of vegetarians reporting having had a PSA test (although general practice records were only available for half of the participants) after adjusting for age differences. Adding PSA screening in the multivariable-adjusted model did not attenuate the estimates, suggesting the differences in PSA screening in vegetarians or fish-eaters in comparison to regular meat-eaters does not explain the observed associations, but other differences in attendance for medical examinations could possibly also contribute. Due to unavailable data in UK Biobank, we were also unable to assess associations by tumour subtypes which may be aetiologically different [35]. Considering the substantial difference in risk we observed for vegetarian men, differences in detection and residual confounding, as well as chance, may contribute to this observed difference.

\section{Interpretation of results: role of confounding and mediation}

The role of residual and unmeasured confounding must be considered when interpreting the findings from this study. Vegetarians and fish-eaters differ from meat-eaters in many non-dietary lifestyle factors such as lower smoking and alcohol consumption, and higher physical activity [52]. Although relevant potential confounders were added to the multivariable models to adjust for these differences, imperfect measurements and/or changes in these confounders over time may result in incomplete adjustment for these variables. For example, the evidence of heterogeneity by smoking status when looking at all cancer as an outcome suggested that residual confounding by smoking may be present.

Differences in BMI between diet groups have also been suggested to explain the lower cancer incidence observed amongst vegetarians [18]; however, when BMI was considered as a potential confounder and mediator, the difference between BMI by diet groups only slightly attenuated the estimates, with the exception of postmenopausal breast cancer. Whether differences in BMI by diet group is due solely to their diet or other lifestyle factors remains unclear, making it difficult to tease out whether BMI mediates or confounds the associations between diet group and cancer risk.

\section{Strengths and limitations}

Strengths of this study include the prospective nature and moderately long follow-up time of participants. Data-linkage to health records was used to determine cancer diagnoses which minimises misclassification and loss to follow-up of participants. The UK Biobank study also gathered data on an array of potential confounders and biochemical biomarkers amongst participants; thus, 
we were able to adjust the models for potential confounding as well as conduct mediation analysis exploring potential mediators between diet groups and cancer risk. When analyses excluded the first 2 years of follow-up, the results remained largely the same, reducing the chance that these associations are due to reverse causality.

There are some limitations to consider in these analyses. Although there were many cancer cases accrued during the follow-up period, these analyses may still be underpowered to detect moderate associations due to the relatively low numbers of cancer cases amongst vegetarians and fish-eaters in this cohort. We also used hospital admissions data to follow-up participants after 2015 in Scotland and 2019 in England because cancer registry data were not available in UK Biobank after this date, which may result in some missing cancer cases and relatively later dates of diagnosis. We also were unable to adjust for total energy intake as this could not be calculated due to the limited number of dietary questions asked at recruitment. As detailed above, the results may be influenced by unmeasured and residual confounding, as well as chance with numerous comparisons, and causality cannot be confirmed. Misclassification of diet may also be possible, as participants may have underreported their intake or changed their diet over the follow-up period, possibly resulting in attenuation of the risk estimates. Vegetarian diets are characterised by not consuming meat, however, this does not necessarily mean that all vegetarians follow a healthy diet, which may influence their risk of cancer and these results. The mediation analyses only explored three potential mediators, and other possible mediating factors such as other biomarkers relevant for cancer, such as oestradiol, were not available. Moreover, baseline BMI and biomarkers were used to assess mediation, and therefore, these measures may not represent BMI during the follow-up and long-term biomarker concentrations, although correlations with repeat measures of BMI and biomarkers showed high agreement [53]. As well, we used the IORW method to explore mediation with bootstrapping CIs, which may make estimates less statistically efficient in comparison to parametric methods, but the IORW has the advantage that it can be applied in survival analysis and provides estimates of the proportion mediated for the mediators of interest. The UK Biobank has been shown to have a healthier risk profile than the UK population [54] and only included British participants most of whom are of white European ancestry (94\%); this may limit generalizability to other populations. However, the risks estimated may still be valid to estimate relative differences for risk-factor disease associations [55].

\section{Conclusions}

In conclusion, this study found that being a low meateater, fish-eater, or vegetarian was associated with a lower risk of all cancer, which may be a result of dietary factors and/or non-dietary differences in lifestyle such as smoking. Low meat-eaters had a lower risk of colorectal cancer, vegetarian women had a lower risk of postmenopausal breast cancer, and men who were vegetarians or fish-eaters had a lower risk of prostate cancer. BMI was found to potentially mediate or confound the association between vegetarian diets and postmenopausal breast cancer. It is not clear if the other associations are causal or a result of differences in detection between diet groups or unmeasured and residual confounding. Future research assessing cancer risk in cohorts with large number of vegetarians is needed to provide more precise estimates of the associations and to explore other possible mechanisms or explanations for the observed differences.

\begin{abstract}
Abbreviations
AHS-2: Adventist Health Study-2; BMI: Body mass index; Cl: Confidence intervals; EPIC-Oxford: European Prospective Investigation into Cancer and Nutrition - Oxford; HES: Hospital episode statistics; HR: Hazard ratio; ICD: International Statistical Classification of Diseases; IGF-I: Insulin-like growth factor-l; IORW: Inverse odds ratio weighting; LRT: Likelihood ratio test; MHT: Menopausal hormone therapy; NDE: Natural direct effect; NIE: Natural indirect effect; NHS: National Health Service; NSAID: Non-steroidal anti-inflammatory drugs; PSA: Prostate-specific antigen; SMR: Scottish Morbidity Records; UK: United Kingdom.
\end{abstract}

\section{Supplementary Information}

The online version contains supplementary material available at https://doi. org/10.1186/s12916-022-02256-w.

Additional File 1: Methods: Covariate classification; Outcome ascertainment; Mediation analysis. Table S1. Baseline characteristics across diet groups separated by sex. Table $\mathbf{S 2}$. Hazard ratio and 95\% confidence intervals for sequential adjustment between association of diet groups and risk of all cancer, colorectal cancer, breast cancer, and prostate cancer. Table S3. Subgroup analyses for diet groups on risk of all cancers. Table S4. Subgroup analyses for diet groups on risk of colorectal cancer. Table S5. Subgroup analyses for diet groups on risk of postmenopausal breast cancer in women. Table S6. Subgroup analyses across diet groups on risk of prostate cancer in men. Table S7. Adjusted and relative means $(95 \% \mathrm{Cl})$ of BMI, IGF-I, and free testosterone concentrations measured at recruitment across diet groups. Fig. S1. Flow diagram of the study exclusion criteria. Fig. S2. Hazard ratios and 95\% confidence intervals for sensitivity analyses including only participants of white ethnicity and complete cases analyses on associations between diet groups and risk of all cancer, prostate, postmenopausal breast, or colorectal cancer. Fig. S3. Hazard ratios and 95\% confidence intervals for sensitivity analyses removing first two years of follow-up and adjusting for fruit and vegetable intake on associations between diet groups and risk of all cancer, prostate, postmenopausal breast, or colorectal cancer.

\section{Acknowledgements}

This work has been conducted using the UK Biobank Resource under Application Number 24494, and we wish to express our gratitude to the participants and those involved in building the resource. UK Biobank is an open access resource. 


\section{Authors' contributions}

CZW, TJK, and AP-C conceived and designed the research question; AP-C managed the project and was responsible for acquiring the data; CZW analysed the data, prepared the figures and tables, and wrote the first draft of the manuscript; CZW prepared the data for analysis; and JAS, YD, TYNT, RKK, AK, RCT, TJK, AP-C provided input on data analysis and interpretation of results. All authors revised the manuscript critically for important intellectual content and read and approved the final manuscript.

\section{Funding}

This work is supported by the Cancer Research UK grant (C8221/A29017) and by the World Cancer Research Fund (WCRF UK), as part of the Word Cancer Research Fund International grant programme (2019/1953). CZW is supported by the Nuffield Department of Population Health Doctor of Philosophy student scholarship. YD is supported by the World Cancer Research Fund UK grant (2019/1953). TYNT is supported by the Nuffield Department of Population Health Intermediate Fellowship and the UK Medical Research Council (MR/M012190/1). RKK is supported by the Clarendon Scholarship from the University of Oxford. AK is supported by the Wellcome Trust, Our Planet Our Health (Livestock, Environment and People - LEAP; 205,212/Z/16/Z). APC is supported by a Cancer Research UK Population Research Fellowship (C60192/ A28516).

\section{Availability of data and materials}

Bona fide researchers can apply to use the UK Biobank dataset by registering and applying at http://ukbiobank.ac.uk/register-apply/.

\section{Declarations}

\section{Ethics approval and consent to participate}

The UK Biobank protocol was approved by the NHS North West Multicentre Research Ethics Committee (21/NW/0157). All participants provided informed consent at recruitment, allowing for follow-up using data-linkage to health records.

\section{Consent for publication}

Not applicable.

\section{Competing interests}

The authors declare no competing interests.

Received: 27 September 2021 Accepted: 13 January 2022

Published online: 24 February 2022

\section{References}

1. Sung H, Ferlay J, Siegel RL, Laversanne M, Soerjomataram I, Jemal A, et al. Global cancer statistics 2020: GLOBOCAN estimates of incidence and mortality worldwide for 36 cancers in 185 countries. CA Cancer J Clin. 2021;71:209-49 https://doi.org/10.3322/caac.21660.

2. Office of the National Statistics. Death registrations summary tables England and Wales. https://www.ons.gov.uk/peoplepopulationandc ommunity/birthsdeathsandmarriages/deaths/datasets/deathregistrati onssummarytablesenglandandwalesreferencetables. Accessed March 15 2021.

3. General Register Office for Scotland. Vital events reference tables. https:// www.nrscotland.gov.uk/statistics-and-data/statistics/statistics-by-theme/ vital-events/general-publications/vital-events-reference-tables. Accessed March 152021

4. Northern Ireland Statistics and Research Agency. Registrar general annual report 2017 cause of death. https://www.nisra.gov.uk/publications/regis trar-general-annual-report-2016-cause-of-death. Accessed 15 Mar 2021

5. Cancer Research UK. Cancer incidence statistics. https://www.cancerrese archuk.org/health-professional/cancer-statistics/incidence/commoncancers-compared.

6. Brown KF, Rumgay H, Dunlop C, Ryan M, Quartly F, Cox A, et al. The fraction of cancer attributable to modifiable risk factors in England, Wales, Scotland, Northern Ireland, and the United Kingdom in 2015. Br J Cancer. 2018;118:1130-41 https://doi.org/10.1038/s41416-018-0029-6.
7. Key TJ, Bradbury KE, Perez-Cornago A, Sinha R, Tsilidis KK, Tsugane S Diet, nutrition, and cancer risk: what do we know and what is the way forward? BMJ. 2020;368:m511 https://doi.org/10.1136/bmj.m511.

8. World Cancer Research Fund/American Institute for Cancer Research. Continuous Update Project Expert Report. Diet, nutrition, physical activity and cancer risk. 2018.

9. Clinton SK, Giovannucci EL, Hursting SD. The World Cancer Research Fund/American Institute for Cancer Research Third Expert Report on Diet, Nutrition, Physical Activity, and Cancer: Impact and Future Directions. J Nutr. 2020;150:663-71 https://doi.org/10.1093/jn/nxz268.

10. Papier K, Tong TY, Appleby PN, Bradbury KE, Fensom GK, Knuppel A, et al. Comparison of major protein-source foods and other food groups in meat-eaters and non-meat-eaters in the EPIC-Oxford cohort. Nutrients. 2019;11:824.

11. Bradbury KE, Tong TYN, Key TJ. Dietary intake of high-protein foods and other major foods in meat-eaters, poultry-eaters, fish-eaters, vegetarians, and vegans in UK Biobank. Nutrients. 2017;9:1317.

12. Key TJ, Appleby PN, Crowe FL, Bradbury KE, Schmidt JA, Travis RC. Cancer in British vegetarians: updated analyses of 4998 incident cancers in a cohort of 32,491 meat eaters, 8612 fish eaters, 18,298 vegetarians, and 2246 vegans. Am J Clin Nutr. 2014;100:378S-85S https://doi.org/10.3945/ ajcn.113.071266.

13. Tantamango-Bartley Y, Jaceldo-Siegl K, Fan J, Fraser G. Vegetarian diets and the incidence of cancer in a low-risk population. Cancer Epidemiol Biomarkers Prev. 2013;22:286-94 https://doi.org/10.1158/1055-9965. Epi-12-1060.

14. Penniecook-Sawyers JA, Jaceldo-Siegl K, Fan J, Beeson L, Knutsen S, Herring $\mathrm{P}$, et al. Vegetarian dietary patterns and the risk of breast cancer in a low-risk population. Br J Nutr. 2016;115:1790-7 https://doi.org/10.1017/ S0007114516000751.

15. Tantamango-Bartley Y, Knutsen SF, Knutsen R, Jacobsen BK, Fan J, Beeson $\mathrm{WL}$, et al. Are strict vegetarians protected against prostate cancer? Am J Clin Nutr. 2015;103:153-60 https://doi.org/10.3945/ajcn.114.106450.

16. Orlich MJ, Singh PN, Sabaté J, Fan J, Sveen L, Bennett H, et al. Vegetarian dietary patterns and the risk of colorectal cancers. JAMA Intern Med. 2015;175:767-76 https://doi.org/10.1001/jamainternmed.2015.59.

17. Cade JE, Taylor EF, Burley VJ, Greenwood DC. Common dietary patterns and risk of breast cancer: analysis from the United Kingdom Women's Cohort Study. Nutr Cancer. 2010;62:300-6 https://doi.org/10.1080/01635 580903441246

18. Appleby PN, Key TJ. The long-term health of vegetarians and vegans. Proc Nutr Soc. 2016:75:287-93 https://doi.org/10.1017/S0029665115004334.

19. Tong TY, Key TJ, Sobiecki JG, Bradbury KE. Anthropometric and physiologic characteristics in white and British Indian vegetarians and nonvegetarians in the UK Biobank. Am J Clin Nutr. 2018;107:909-20 https://doi. org/10.1093/ajen/nqy042.

20. Rosell M, Appleby P, Spencer E, Key T. Weight gain over 5 years in 21966 meat-eating, fish-eating, vegetarian, and vegan men and women in EPICOxford. Int J Obes. 2006;30:1389-96 https://doi.org/10.1038/sj.ijo.08033 05 .

21. Tong TYN, Perez-Cornago A, Bradbury KE, Key TJ. Biomarker concentrations in white and British Indian vegetarians and non-vegetarians in the UK Biobank. J Nutr. 2021; https://doi.org/10.1093/jn/nxab192.

22. Allen NE, Appleby PN, Davey GK, Key TJ. Hormones and diet: Iow insulinlike growth factor-l but normal bioavailable androgens in vegan men. $\mathrm{Br} J$ Cancer. 2000;83:95-7 https://doi.org/10.1054/bjoc.2000.1152.

23. Allen NE, Appleby PN, Davey GK, Kaaks R, Rinaldi S, Key TJ. The associations of diet with serum insulin-like growth factor I and its main binding proteins in 292 women meat-eaters, vegetarians, and vegans. Cancer Epidemiol Biomarkers Prev. 2002;11:1441-8.

24. Knuppel A, Fensom GK, Watts EL, Gunter MJ, Murphy N, Papier K, et al. Circulating insulin-like growth factor-I concentrations and risk of 30 cancers: prospective analyses in UK Biobank. Cancer Res. 2020;80:4014-21 https:// doi.org/10.1158/0008-5472.Can-20-1281.

25. Watts EL, Fensom GK, Smith Byrne K, Perez-Cornago A, Allen NE, Knuppel A, et al. Circulating insulin-like growth factor-l, total and free testosterone concentrations and prostate cancer risk in 200000 men in UK Biobank. Int J Cancer. 2021;148:2274-88 https://doi.org/10.1002/ijc.33416.

26. Tin Tin S, Reeves GK, Key TJ. Endogenous hormones and risk of invasive breast cancer in pre- and post-menopausal women: findings from 
the UK Biobank. Br J Cancer. 2021;125:126-34 https://doi.org/10.1038/ s41416-021-01392-z.

27. UK Biobank. Background. https://www.ukbiobank.ac.uk/learn-moreabout-uk-biobank/about-us. Accessed.

28. Vermeulen A, Verdonck L, Kaufman JM. A critical evaluation of simple methods for the estimation of free testosterone in serum. J Clin Endocrinol Metab. 1999;84:3666-72 https://doi.org/10.1210/jcem.84.10.6079.

29. UK Biobank. Analysis of biological samples. https://www.ukbiobank.ac. uk/enable-your-research/about-our-data/biomarker-data. Accessed 25 Mar 2021.

30. UK Biobank. Cancer data: linkage from national cancer registries https:// biobank.ndph.ox.ac.uk/crystal/crystal/docs/CancerLinkage.pdf. Accessed 15 Sept 2020.

31. Collaborative Group on Hormonal Factors in Breast Cancer. Menarche, menopause, and breast cancer risk: individual participant meta-analysis, including 118964 women with breast cancer from 117 epidemiological studies. Lancet Oncol. 2012;13:1141-51 https://doi.org/10.1016/ S1470-2045(12)70425-4

32. Perez-Cornago A, Key TJ, Allen NE, Fensom GK, Bradbury KE, Martin RM, et al. Prospective investigation of risk factors for prostate cancer in the UK Biobank cohort study. Br J Cancer. 2017;117:1562-71 https://doi.org/10. 1038/bjc.2017.312.

33. Parish S, Peto R, Palmer A, Clarke R, Lewington S, Offer A, et al. The joint effects of apolipoprotein B, apolipoprotein A1, LDL cholesterol, and HDL cholesterol on risk: 3510 cases of acute myocardial infarction and 9805 controls. Eur Heart J. 2009;30:2137-46 https://doi.org/10.1093/eurheartj/ ehp221.

34. Floud S, Balkwill A, Moser K, Reeves GK, Green J, Beral V, et al. The role of health-related behavioural factors in accounting for inequalities in coronary heart disease risk by education and area deprivation: prospective study of 1.2 million UK women. BMC Medicine. 2016;14:145. https://doi. org/10.1186/s12916-016-0687-2

35. Perez-Cornago A, Appleby PN, Pischon T, Tsilidis KK, Tiønneland A, Olsen $A$, et al. Tall height and obesity are associated with an increased risk of aggressive prostate cancer: results from the EPIC cohort study. BMC Medicine. 2017;15:115 https://doi.org/10.1186/s12916-017-0876-7.

36. Tchetgen Tchetgen EJ. Inverse odds ratio-weighted estimation for causal mediation analysis. Stat Med. 2013;32:4567-80 https://doi.org/10.1002/ sim. 5864

37. Nguyen QC, OsypukTL, Schmidt NM, Glymour MM, Tchetgen Tchetgen EJ. Practical guidance for conducting mediation analysis with multiple mediators using inverse odds ratio weighting. Am J Epidemiol. 2015;181:349-56 https://doi.org/10.1093/aje/kwu278.

38. VanderWeele TJ, Vansteelandt S. Mediation analysis with multiple mediators. Epidemiol Methods. 2014;2:95-115 https://doi.org/10.1515/ em-2012-0010.

39. VanderWeele TJ. Mediation analysis: a practitioner's guide. Annu Rev Public Health. 2016;37:17-32 https://doi.org/10.1146/annurev-publh ealth-032315-021402.

40. Bouvard V, Loomis D, Guyton KZ, Grosse Y, Ghissassi FE, Benbrahim-Tallaa $L$, et al. Carcinogenicity of consumption of red and processed meat. Lancet Oncol. 2015;16:1599-600 https://doi.org/10.1016/S1470-2045(15) 00444-1.

41. World Cancer Research Fund/American Institute for Cancer Research. Continuous Update Project Expert Report. Diet, nutrition, physical activity and colorectal cancer. 2018.

42. Bradbury KE, Murphy N, Key TJ. Diet and colorectal cancer in UK Biobank: a prospective study. Int J Epidemiol. 2019;49:246-58 https://doi.org/10. 1093/ije/dyz064.

43. Lauby-Secretan B, Scoccianti C, Loomis D, Grosse Y, Bianchini F, Straif K. Body fatness and cancer-viewpoint of the IARC working group. N Engl J Med. 2016;375:794-8 https://doi.org/10.1056/NEJMsr1606602.

44. Ortega LS, Bradbury KE, Cross AJ, Morris JS, Gunter MJ, Murphy N. A prospective investigation of body size, body fat composition and colorectal cancer risk in the UK Biobank. Sci Rep. 2017;7:17807 https://doi.org/10. 1038/s41598-017-17997-5.

45. Gilsing AM, Weijenberg MP, Goldbohm RA, Dagnelie PC, van den Brandt $\mathrm{PA}$, Schouten $\mathrm{L}$. Vegetarianism, low meat consumption and the risk of lung, postmenopausal breast and prostate cancer in a population-based cohort study. Eur J Clin Nutr. 2016;70:723-9 https://doi.org/10.1038/ejcn. 2016.25 .
46. World Cancer Research Fund/American Institute for Cancer Research Continuous Update Project Expert Report. Diet, nutrition, physical activity and breast cancer. 2018.

47. Tong TYN, Appleby PN, Bradbury KE, Key TJ. Cross-sectional analyses of participation in cancer screening and use of hormone replacement therapy and medications in meat eaters and vegetarians: the EPICOxford study. BMJ Open. 2017;7:e018245 https://doi.org/10.1136/bmjop en-2017-018245.

48. World Cancer Research Fund/American Institute for Cancer Research. Continuous Update Project Expert Report. Diet, nutrition, physical activity and prostate cancer. 2018.

49. Watling C, Kelly RK, Tong TYN, Piernas C, Watts EL, TinTin S, et al. Associations of circulating insulin-like growth factor-I with intake of dietary proteins and other macronutrients. Clin Nutr. 2021;40:4685-93. https:// doi.org/10.1016/j.clnu.2021.04.021.

50. Travis RC, Appleby PN, Martin RM, Holly JMP, Albanes D, Black A, et al. A meta-analysis of individual participant data reveals an association between circulating levels of IGF-I and prostate cancer risk. Cancer Res. 2016;76:2288-300 https://doi.org/10.1158/0008-5472.CAN-15-1551.

51. Ibrayev Y, Oda K, Fraser GE, Knutsen SF. Utilization of prostate cancer screening according to dietary patterns and other demographic variables. The adventist health study-2. J Cancer. 2013;4:416-26 https://doi. org/10.7150/jca.6442.

52. Davey GK, Spencer EA, Appleby PN, Allen NE, Knox KH, Key TJ. EPICOxford:lifestyle characteristics and nutrient intakes in a cohort of 33883 meat-eaters and 31546 non meat-eaters in the UK. Public Health Nutr. 2003;6:259-68 https://doi.org/10.1079/PHN2002430.

53. Allen N, Arnold M, Parish S, Hill M, Sheard S, Callen H, et al. Approaches to minimising the epidemiological impact of sources of systematic and random variation that may affect biochemistry assay data in UK Biobank. Wellcome Open Res. 2021;5 https://doi.org/10.12688/wellcomeopenres. 16171.2.

54. Fry A, Littlejohns TJ, Sudlow C, Doherty N, Adamska L, Sprosen T, et al. Comparison of sociodemographic and health-related characteristics of UK Biobank participants with those of the general population. Am J Epidemiol. 2017;186:1026-34 https://doi.org/10.1093/aje/kw×246.

55. Batty GD, Gale CR, Kivimäki M, Deary IJ, Bell S. Comparison of risk factor associations in UK Biobank against representative, general population based studies with conventional response rates: prospective cohort study and individual participant meta-analysis. BMJ. 2020;368:m131 https://doi.org/10.1136/bmj.m131.

\section{Publisher's Note}

Springer Nature remains neutral with regard to jurisdictional claims in published maps and institutional affiliations.

Ready to submit your research? Choose BMC and benefit from:

- fast, convenient online submission

- thorough peer review by experienced researchers in your field

- rapid publication on acceptance

- support for research data, including large and complex data types

- gold Open Access which fosters wider collaboration and increased citations

- maximum visibility for your research: over $100 \mathrm{M}$ website views per year

At BMC, research is always in progress.

Learn more biomedcentral.com/submissions 